\title{
Improvement of Color and Luminance Uniformity of the Edge-Lit Backlight Using the RGB LEDs
}

\author{
Chang Gyun Son, Jong Hoon Yi, Jin Seog Gwag, and Jin Hyuk Kwon* \\ Department of Physics, Yeungnam University, Gyeongsan 712-749, Korea \\ Gyeungju Park \\ Department of Optometry, Sunlin University, Pohang 791-712, Korea
}

(Received May 13, 2011 : revised June 15, 2011 : accepted June 15, 2011)

\begin{abstract}
The effect of the M-window color mixing bar on the characteristics of color mixing and hot spots in the edge-lit backlight employing red $(R)$, green $(G)$, blue $(G)$ light emitting diodes (LED) were studied in terms of the structure of the M-window color mixing bar. The rays from RGB LEDs entering the $\mathrm{M}$-window bar were mixed by internal reflection and scattering inside the M-window bar so that the hot spots and color separation were minimized. The M-window bar was designed and fabricated and the simulation results are matched quite well to experimental data.
\end{abstract}

Keywords : Display, LED, Hot spot, Color separation

OCIS codes : (120.2040) Displays; (230.3720) Liquid crystal devices; (330.1690) Color

\section{INTRODUCTION}

Recently the light emitting diodes (LED) became the major light sources for the edge-lit liquid crystal display (LCD) backlight because the color gamut of the white light LEDs became wide enough to compete the cold-cathode fluorescent lamp (CCFL), and the white LEDs are free from the colormixing problem. So, the white LEDs are being used in the large-size LCDs such as LCD TVs as well as the smallsize LCDs such as the mobile and notebook [1-4]. The hot spot problem in the LED backlight unit (BLU) is not so serious when the white LEDs are used as the light sources because many low power white LEDs are placed very close to each other [5]. However, the future trend of the LCD will be LCDs having high image quality in order to compete with the organic light emitting diode (OLED) that is rising as a strong competitor to the LCD. In order to achieve the high image quality with color gamut exceeding $100 \%$ of NTSC, two different approaches are possible. One is developing the white LED that has a wider color gamut by using three or more phosphor materials, and the other is using RGB LEDs as the light sources. Typically the WLEDs have smaller color gamut due to the lack of the red spectral region when a yellow phosphor is used to convert the blue light from the blue LED. The phosphor materials for wide color gamut WLED are still under development [6]. Also, WLEDs show poor white balance in terms of the emission angle because the thickness of phosphors does not change exactly in the same angular profile of the blue light intensity [7].

However, RGB LEDs are already well developed light sources and the challenge is how to design the backlight system free from any hot spots or color nonuniformity through sufficient color mixing $[8,9]$. A typical structure of the edge-lit BLU as shown in Fig. 1 is composed of the light sources, reflector, light guide panel (LGP), the diffuser sheet (DS), the prism sheet, and the reflective polarizer sheet $[10,11]$. Lights coming out of the RGB LEDs are mixed by total internal reflection and scattering in the lightguide panel to produce the white color. When there is no color-mixing device in front of the lightguide panel, the hot spots and the hot lines at the corresponding positions of LEDs appear as shown in Fig. 2 since the straight lights from LEDs have more intensity than other directions [12]. Also, the simulation shows that the color spread becomes as large as $\Delta x=0.2, \Delta y=0.28$ in the CIE chromati-

\footnotetext{
*Corresponding author: jhkwon@ynu.ac.kr

Color versions of one or more of the figures in this paper are available online.
} 




FIG. 1. The structure of the edge-lit backlight unit including the reflector, light guide panel, diffuser sheet, prism sheet, reflective polarizer sheet, and red, green, blue light emitting diodes (LED).

city diagram when there in no color mixer. This shows that when RGB LEDs are used as the light sources the color separation as well as the hot spots will be another serious problem. Especially at the side region where a special color LED is located the white balance is very hard to achieve [13].

In this paper the problems of the hot spots, hot lines, and color nonuniformity are solved by inserting a color mixing bar that has M-type coupling windows between the lightguide panel and the RGB LEDs. Simulations of using the color mixing bar are presented and the experimental result shows that it is very efficient in eliminating the hot spots, hot lines, and color nonuniformity.

\section{SIMULATION}

The simulation model of the edge-lit LED BLU is composed of a transparent PMMA light guide panel with size $3 \times 184.2 \times 117.9 \mathrm{~mm}^{3}$, a diffuser sheet, a prism sheet, and a reflective polarizer sheet as shown in Fig. 1. The diffuser sheet has a diffusing layer on the top surface of the surface with a Gaussian scattering property with spread angle of 15 degrees. The prism sheet has the micro prism array on the top surface of the sheet with prism structure of 50 microns in pitch and apex angle of 90 degrees. The reflective polarizer film was set with transmittance of $90 \%$ and $10 \%$ for the two orthogonal polarizations. 30 RGB LEDs are placed in groups of RGB LED with $10 \mathrm{~mm}$ between the adjacent LEDs. The size of LED chips are given as $0.1 \times 0.4 \times 0.4 \mathrm{~mm}^{3}$ and the output flux of RGB LEDs are $36,250,15$ lumens with emission wavelengths of 620,570 , and $465 \mathrm{~nm}$, respectively.

First, the simulation was performed for the conventional backlight structure without any color-mixing bar. Fig. 2 shows a typical simulation results for the illuminance chart, CIE chromaticity diagram for the color distribution, and the vertical (dashed line) and horizontal (solid line) angular luminance profiles in polar coordinates. The illuminance chart shows the strong hot spots in front of the LED groups, and the wide color spread $\Delta x=0.2, \Delta y=0.28$ in the chromaticity diagram shows that the color mixing is very poor. Fig. 3 shows the change of the strength of the hot spots as the gap between the LEDs and the lightguide panel was varied from $0.5 \mathrm{~mm}$ to $3.5 \mathrm{~mm}$. When the gap was set as

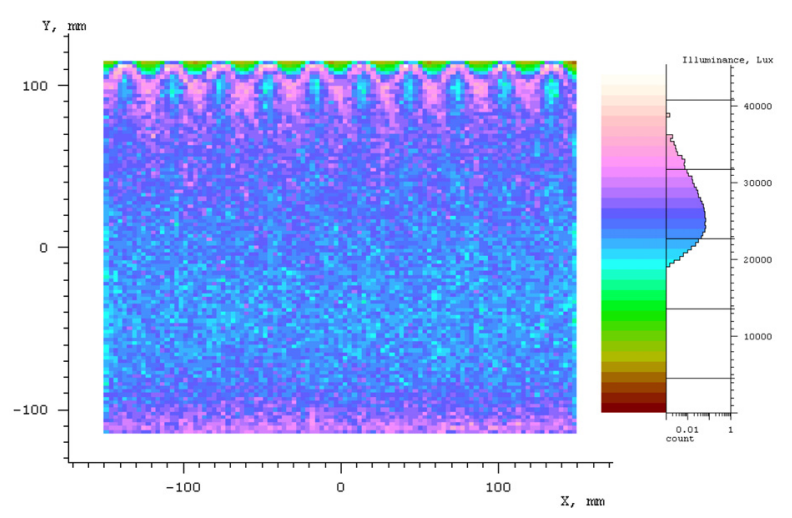

(a)



(b)

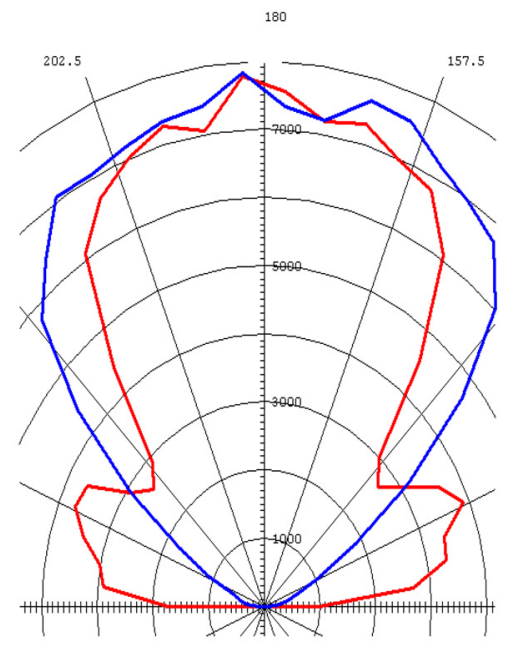

(c)

FIG. 2. Simulation results of RGB LED BLU without color mixing bar. (a) Hot spots appear at the right edge of the lightguide panel of the conventional edge-lit BLU using RGB LEDs, (b) the color distribution in the CIE chromaticity diagram showing color spread $\Delta x=0.2, \Delta y=0.28$.

$0.5 \mathrm{~mm}$, strong hot spots appeared as shown in Fig. 3(a). As the gap was increased up to $3.5 \mathrm{~mm}$, the hot spots became weak, but the color mixing is still very poor as 

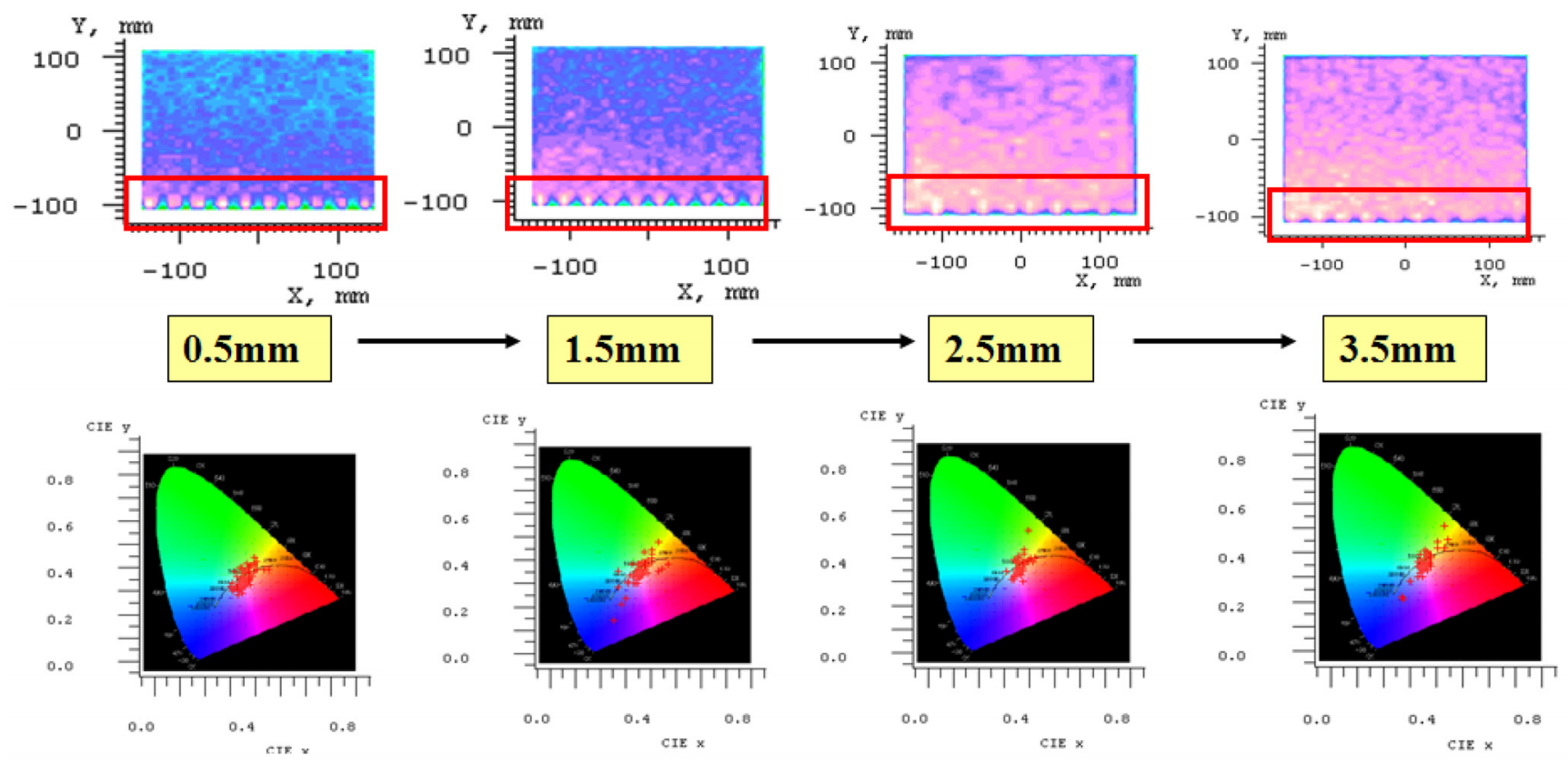

FIG. 3. The hot spots and the color spreads in the chromaticity diagram in terms of the gap between LEDs and light guide panel.

shown in the chromaticity diagram of Fig. 3(d).

Fig. 4 shows various types of color mixing bar such as the lenticular coupling type and the prism coupling type $[14,15]$. In both cases the problems of hot spots and color mixing are not solved perfectly. Fig. 5 shows the proposed edge-lit backlight system with M-window color-mixing bar placed between the lightguide panel and the RGB LEDs. The RGB lights from the RGB LEDs enters the color mixing bar by the M-window reflecting facets and then they are mixed to generate white light by the total internal reflection and scattering in wide range covering several LEDs. The size of the M-window bar was $3 \times 4 \times 187.5 \mathrm{~mm}$ and the M-window has entrance width $2 \mathrm{~mm}$ and depth $1 \mathrm{~mm}$. The slanted surface of the M-window was coated with a specular reflecting layer and the side surfaces except the exit surface were treated to be reflective with reflectance of $98 \%$. Fig. 6 shows the simulation results of the backlight system when the M-window color-mixing bar was placed between the lightguide panel and the RGB LEDs. It is seen in illuminance chart of Fig. 6(a) that most of the hot spots disappeared and the CIE chromaticity diagram shows that the color distribution on the backlight is centered around the white spot showing the color spreading $\Delta x=0.06, \Delta y$ $=0.13$ and this means that the color mixing is very good. Also, the angular luminance profile of Fig. 6(c) shows the view angle is $104 \times 80$ degrees along the horizontal and the vertical directions.

Table 1 shows the simulation results for the various coupling types such as the bare surface (no color mixer), the lenticular lens type shown in Fig. 4 (a), the prism coupling type shown in Fig. 4(b), and the M-window bar. The contrast ratio CR and the hot spot ratio HSR measured at $5 \mathrm{~mm}$ apart from the entrance surface of the lightguide
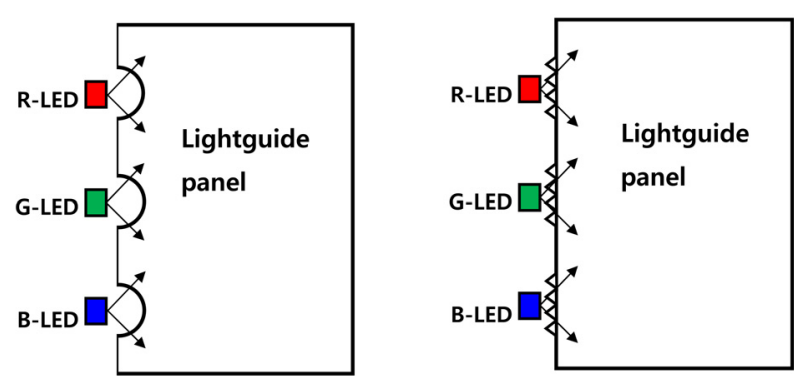

FIG. 4. The color mixing bar with textures of (a) lenticular lens type and (b) prism coupling type.

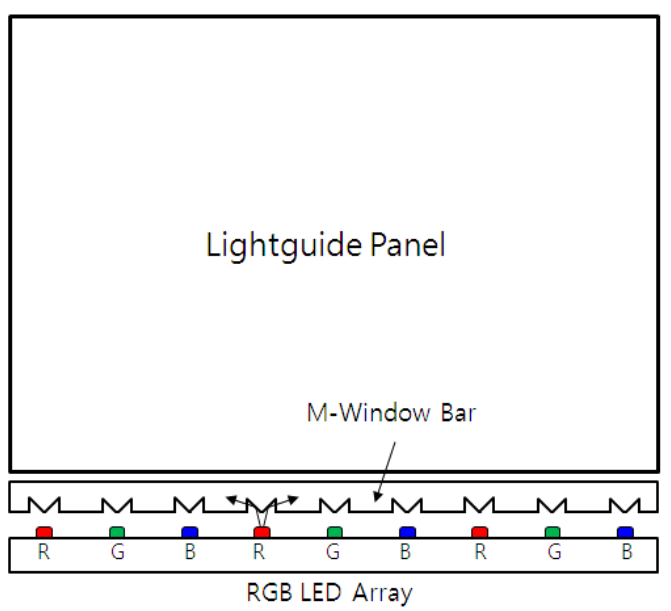

FIG. 5. A schematic of the M-window color-mixing bar inserted between RGB LEDs and light guide panel. The light from RGB LEDs are reflected and coupled into the M-window bar so that they are mixed by the total internal reflection and scattering before they enter the lightguide panel. 


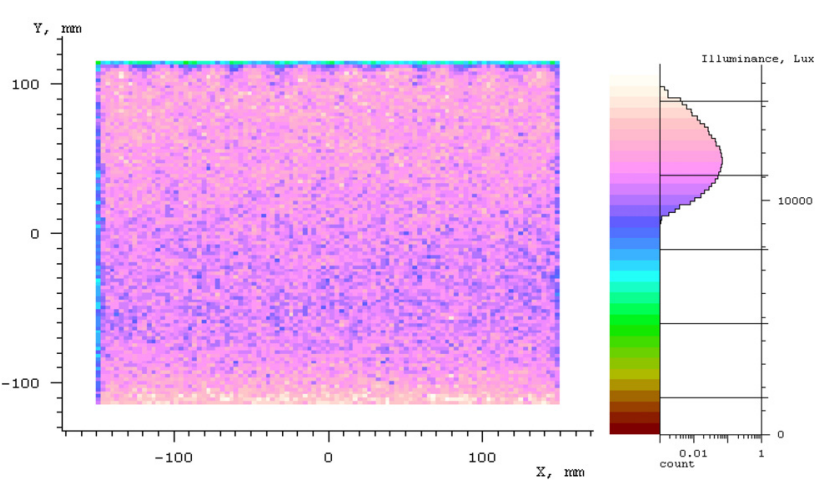

(a)

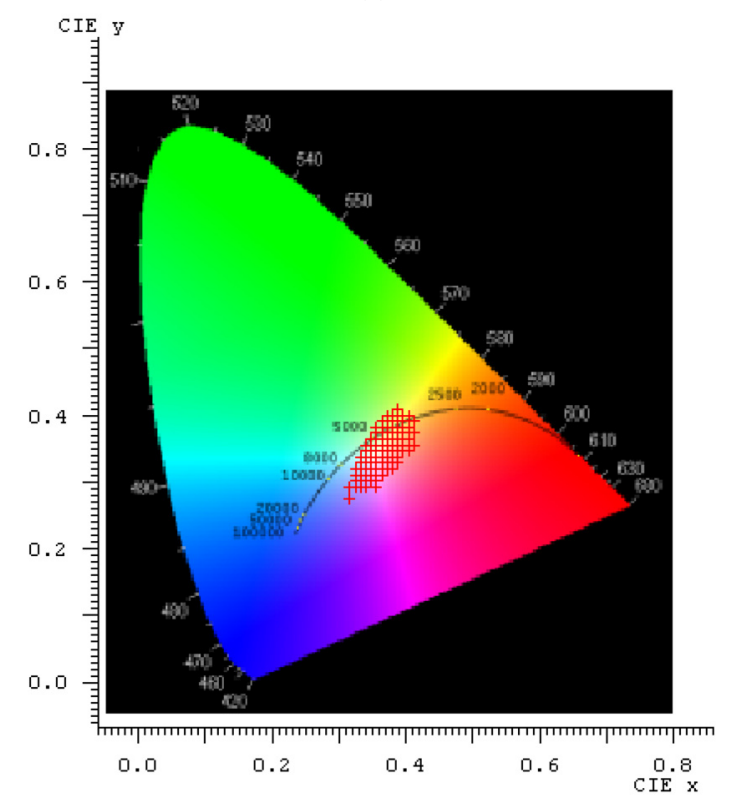

(b)

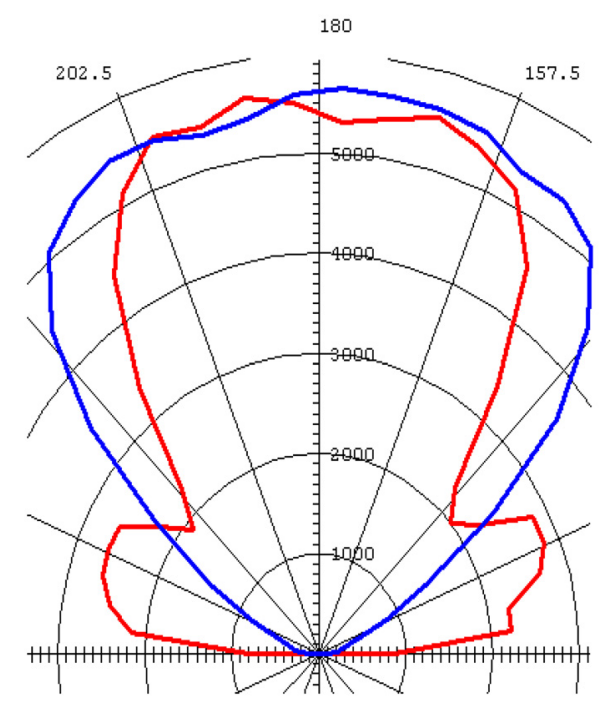

(c)

FIG. 6. Simulation results of RGB LED BLU with the color mixing bar. (a) the illuminance chart shows that the hot spots disappeared, (b) the color distribution in the CIE chromaticity diagram showing color spread $\Delta x=0.06, \Delta y=0.13$.

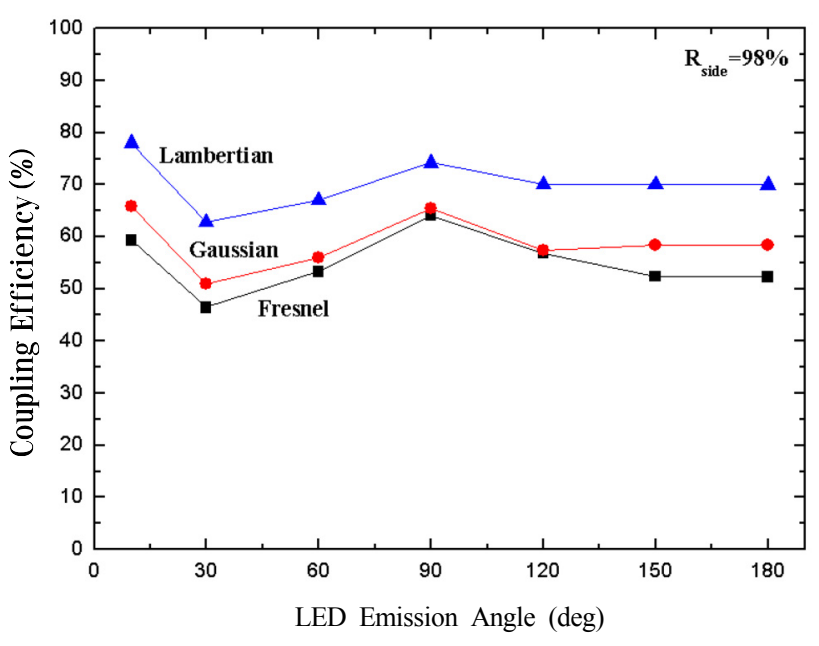

FIG. 7. The simulation results of the coupling efficiency in terms of the LED emission angle, the optical property of the exit surface.

panel are introduced for comparative purpose as follows,

$$
\begin{aligned}
& C R=\frac{I_{\max }-I_{\min }}{I_{\max }+I_{\min }}, \\
& H S R=\frac{I_{\max }}{I_{\min }},
\end{aligned}
$$

where $I_{\max }$ and $I_{\min }$ are the maximum and minimum values of the hot spot illuminance. CR value ranges between 0 and 1 , and the HSR value is given larger than one. Table 1 shows that the CR and HSR values of the bare surface coupling, lenticular lens type coupling, and the prism type coupling show similar values and this means that these couplings from the LEDs into the lightguide panel contribute very little to the improvement of the hot spot and color nonuniformity. On the contrary, the CR and HSR values for the M-window bar coupling shows more than 3 times more improvement compared to the other coupling types.

The insertion of the M-window color mixing bar between the RGB LEDs and the lightguide panel tend to induce the loss of light power that is coupled into the lightguide panel. The major parameters affecting the coupling efficiency that is defined as the ratio of light power coupled into the lightguide panel compared to the total output power of LED will be the emission angle of LEDs, optical property of side surfaces of the M-window bar and the exit surface. Fig. 7 shows the change of the coupling efficiency in terms of the LED emission angle and the optical property of the exit surface. The LED emission angle was changed from 10 degree to 180 degree in steps of 30 degrees and the optical property of the exit surface were assumed to be Fresnel, Gaussian, and Lambertian scattering surfaces. The reflectance and transmittance of the Gaussian and Lambertian scattering 
surfaces were assumed to be $10 \%$ and $90 \%$, respectively. The optical property of the side surfaces of the M-window bar was assumed to have the Lambertian reflectivity of 98\%. Fig. 7 shows the simulation results of the coupling efficiency in terms of the LED emission angle and the optical property of the exit surface. The Lambertian exit surface shows the better coupling efficiency compared to the Gaussian and Fresnel surfaces and the LED emission angles of 10 and 90 degrees show larger coupling efficiency compared to the other LED emission angles. The higher coupling efficiency of the Lambertian exit surface is thought to come from the shorter propagation length of light through the M-window bar. In contrast, the mixing effect of light inside the M-window bar for the Fresnel exit surface will be the best among the three types of the exit surface.

\section{EXPERIMENTS}

A prototype M-window color-mixing bar was fabricated by machining a polycarbonate bar as shown in Fig. 8. The size of the bar was $3 \times 4 \times 187.5 \mathrm{~mm}^{3}$ and the size of the M-window was $2 \mathrm{~mm}$ in the entrance window and $1 \mathrm{~mm}$ in depth. The slanted surfaces were coated with a reflecting layer of reflectivity $98 \%$. The M-window color-mixing bar was inserted between the RGB LEDs and the lightguide panel. The observed hot spots are almost disappeared as shown in Fig. 9(a) compared to the strong hot spots shown

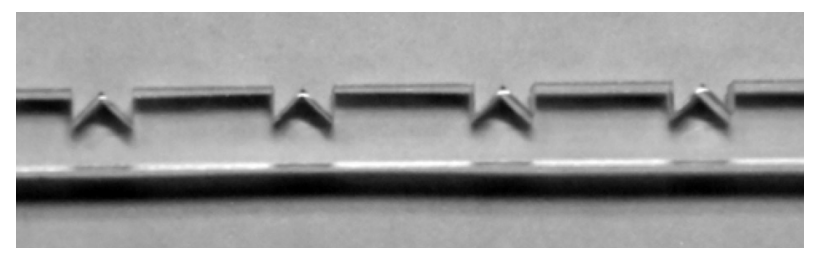

FIG. 8. The fabricated M-window color mixing bar. The size is $3 \times 4 \times 187.5 \mathrm{~mm}^{3}$.

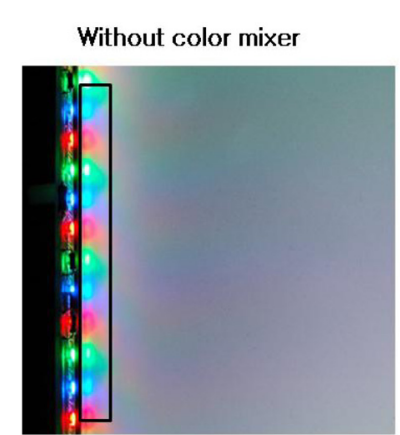

(a)

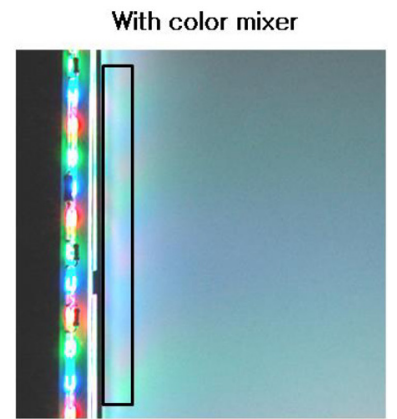

(b)
FIG. 9. Hot spots of RGB LED backlight. (a) shows the strong hot spots without the M-window color-mixing bar, and (b) shows that most of the hot spots disappeared when the M-window color-mixing bar was inserted between the RGB LED and the lightguide panel.



FIG. 10. Power variation of the hot spots in terms of the position along the entrance surface of the lightguide panel.

in Fig. 9(b). Fig. 10 shows the measured power in terms of the position along the entrance surface of the lightguide. When there is no color mixing bar, strong peaks appear exactly at the corresponding positions of the RGB LEDs, however, the power becomes uniform when the M-window color mixing bar war inserted between the LEDs and the lightguide panel. The maximum to minimum power ratios are 16.7 and 1.3 without and with the color-mixing bar. This means that the M-window color-mixing bar is as efficient as about 12.8 times in reducing the hot spots.

\section{CONCLUSION}

The edge-lit RGB LED backlight has serious problems such as the hot spots and color nonuniformity. We designed the M-window color mixing bar that can eliminate efficiently the hot spots and color nonuniformity in the RGB LED backlight. The simulation and experimental results showed that the color mixing bar with $\mathrm{M}$-windows works very well. In the conventional backlight system with RGB LEDs, the hot spots disappeared when the distance between RGB LED and the entrance surface of the lightguide panel was larger than $3.5 \mathrm{~mm}$, but the color mixing was still poor. This means that it is very difficult to eliminate both the hot spots and color nonuniformity at the same time in the conventional edge-lit RGB LED backlight system. However, it is shown in this paper that both the hot spots and the color nonuniformity can be solved together by introducing the M-window color-mixing bar between the RGB LEDs and the lightguide panel. The introduction of an M-window color-mixing bar tends to reduce the normal luminance due to loss of light energy in the color-mixing bar and more research is needed to optimize the structure of the colormixing bar the size of the M-window, the reflectance, and the optical scattering properties of the color mixing bar. The loss of light can be compensated by reduction of LED 
number because the high power LEDs can be used with larger spacing between the adjacent LEDs. The optimum spacing between adjacent LEDs and the total LED number will be determined by the LCD size, the total output flux of the LCD, and the output power of the LEDs.

\section{ACKNOWLEDGMENT}

This research was supported by the Yeungnam University research grant in 2009.

\section{REFERENCES}

1. K. Kakinuma, "Technology of wide color gamut backlight with light-emitting diode for liquid crystal display television," Jpn. J. Appl. Phys. 45, 4330-4334 (2006).

2. J.-H. Ko, "Recent research trends in development of new light sources for the backlight unit of liquid crystal display," Asian J. Phys. 14, 231-237 (2005).

3. I. Kim and K. Chung, "Wide color gamut backlight form three-band white LED," J. Opt. Soc. Korea 11, 67-70 (2007).

4. S.-T. Hur and S.-W. Choi, "An edge-lit backlight unit for small portable liquid crystal displays with a newly designed single pyramid sheet," J. Korean Phys. Soc. 58, 392-395 (2011).

5. B. Kim, J. Kim, W.-S. Ohm, and S. Kang, "Eliminating hotspots in a multi-chip LED array direct backlight system with optimal patterned reflectors for uniform illuminance and minimal system thickness," Opt. Express 18, 8595-8604 (2010).

6. H. Y. Ryu and D. H. Kim, "High-brightness phosphor-conversion white light source using InGaN blue laser diode," J. Opt. Soc. Korea 14, 415-419 (2010).

7. J. K. Sheu, S. J. Chang, C. H. Kuo, and Y. K. Su, L. W. Wu, Y. C. Lin, W. C. Lai, J. M. Tsai, G. C. Chi, and R. $\mathrm{K}$. Wu, "White-light emission from near UV InGaN-GaN LED chip precoated with blue/green/red phosphors," IEEE Photon. Technol. Lett. 15, 18-20 (2003).

8. R. S. West, G. D. Gray, and J. W. Stewart, "Side emitting light emitting device," Lumiled Lighting, U. S. Patent 6598998 (2003).

9. Y.-H. Lu and C.-H. Tien, "A novel direct-led-backlight unit using grooved hexagonal light-guide plate," in Proc. SID '06 Digest (San Francisco, USA, Jun. 2006), pp. 1513-1516.

10. G. Park, T. S. Aum, and J. H. Kwon, "Characterization and modeling light scattering in diffuser sheets," J. Korean Phys. Soc. 54, 44-48 (2009).

11. S. Kobayashi, S. Mikoshiba, and S. K. Lim, LCD Backlights (John Wiley \& Sons Inc., California, USA, 2009), Chapter 14.

12. C. M. Ju, "Light module for LCD panel," U. S. Patent 6871973 (2005).

13. W. Folkerts, "LED backlighting concepts with high flux LEDs," in Proc. SID '04 Digest (Seattle, USA, May 2004), pp. 1226-1229.

14. G. T. Lee, "Backlight unit," Korean Patent 2006-0028895 (2006).

15. J. H. Min, M. G. Lee, S. M. Lee, J. H. Kim, and J. S. Choi, "Backlight Unit," U. S. Patent 6,979,095 (2005). 